
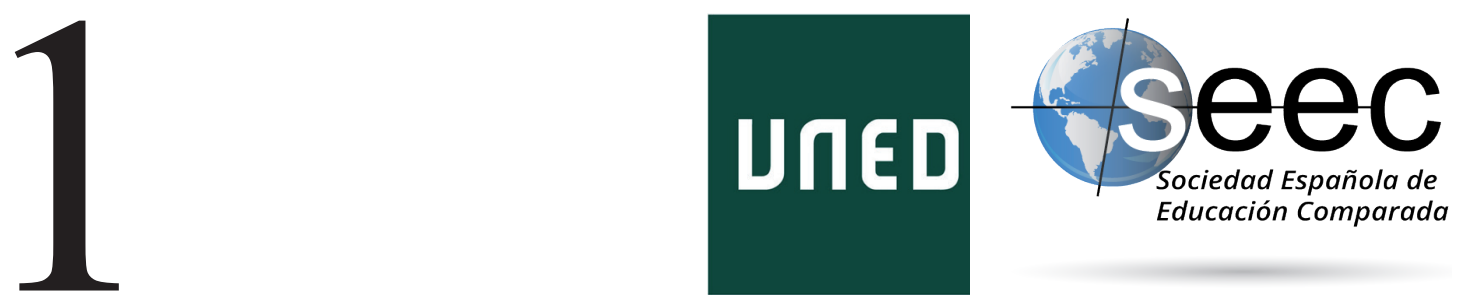

\title{
Editorial: Comparatística y Metodología
}

\section{Editorial: Comparatistic and Methodology}

\section{Pedro Aullón de Haro*}

DOI: $10.5944 /$ reec.34.2019.25082

\section{Recibido: 3 de mayo de 2019 Aceptado: 21 de mayo de 2019}

\footnotetext{
* Pedro Aullón de Haro: Doctor en Filosofía y Letras, catedrático de Teoría de la Literatura y Literatura Comparada. Es ideador de una Estética general, así como teórico del Ensayo, de la poesía moderna y la Historia de las Ideas; impulsor de los estudios de Asia en España y de la epistemología de las Ciencias humanas y la Ciencia de la literatura. Ha desarrollado intelectual y académicamente las ideas y conceptos mayores de «universalidad, sublimidad y globalización», «humanismo universal», «Convergencia Ética», «universalidad barroca», «ideación», «continuidad estética», "estética de la lectura», «filipinismo», «géneros ensayísticos», «discurso reflexivo», «malversación intelectual», «escatología y final de la Crítica», «metodologías comparatistas», «metodologías humanísticas en la Era digital». Creó y dirige desde 1994, en la Universidad de Alicante, el «Grupo de Investigación Humanismo-Europa» que otorga el «Premio Juan Andrés de Ensayo e Investigación en Ciencias Humanas», y de otra parte el «Instituto Juan Andrés de Comparatística y Globalización». En la actualidad asimismo dirige las colecciones «Mayor» y «Menor» de Editorial Verbum. Datos de contacto: E-mail: p.aullondeharo@gmail.com
} 
Con toda probabilidad, el mayor problema del pensamiento epistemológico moderno consiste en la carencia de una Epistemología trazada y estable de las Ciencias humanas como conjunto, justamente a diferencia del caso de las Ciencias físico-naturales. No me propongo entrar ahora en ese gran asunto general de extraordinaria repercusión, pero sí en algo que en realidad profundamente le atañe, es decir uno de los fenómenos más sorprendentes de la entidad y evolución de la Epistemología moderna. Me refiero a la cuestión relativa al Comparatismo, a las Metodologías comparatistas o a la Comparatística en general, según se prefiera subrayar mediante una u otra denominación un distinto aspecto o sector de la gama de procedimientos disciplinares consistente en la focalización o el ejercicio de la comparación entre elementos susceptibles de ser sometidos a relación contrastiva rentable (dejando ahora al margen las particularizaciones sectoriales que definen los diferentes campos disciplinares).

A este propósito sería necesario constatar de momento, i) la evidencia de que ese tipo de procedimiento es habitual, de manera manifiesta o presupuesta, en todo campo disciplinar, ya físico-natural o humanístico; ii) esto es así en justa correspondencia, o inherencia, con el hecho de que dicho tipo de procedimiento es reconocidamente un modo de comportamiento común no científico sino del natural funcionamiento o expresión del psiquismo del ser humano; iii) se trata de un procedimiento metodológico surgido en la antigüedad grecolatina, ejercido en los campos físico-naturales, como es el caso que devendrá Anatomía comparada, al igual que en los campos humanísticos de más alcance, sobre la base del «parangón» cultural grecolatino pero, técnicamente sobre todo, mediante la perfilación de un estricto método a manos de ese gran virtuoso intelectual, Dionisio de Halicarnaso, padre o primer gran maestro de la Crítica literaria propiamente dicha, que se aplicó al debate crítico de la relación interna del texto platónico y de este con otros textos importantes. Me refiero al tratadito epistolar titulado Carta a Pompeyo Gémino. Acaso esto no cabía fuese de otro modo, pues no se adelantó Aristóteles en ese cometido teórico, más atento que otra cosa el Estagirita a la capacidad constructiva de la techne, cuando menos en lo que se refiere a los objetos verbales, de primer rango para la ideación griega, es decir la Retórica y la Poética.

El asunto consiste (lo reformularé simplificadamente y de nuevo) en que la comparación, el comparatismo como procedimiento mental constituye una reconocida forma fundamental y esencial de relación y reacción del psiquismo humano en el mundo (no siendo necesario entretener aquí argumentos sobre esto último, aunque no dejaría de ser ilustrativo); el comparatismo como procedimiento de relaciones culturales no es sino la creación greco-latina del «parangón» tipificado en la relación Homero/Virgilio y más tarde Homero/la Biblia; el comparatismo como método es ideación de la Crítica literaria de Dionisio de Halicarnaso, según ha quedado dicho; el comparatismo como procedimiento disciplinar extenso y universalista no es sino la culminación de la Historia universal de las ciencias y las letras eminentemente por Juan Andrés, así como la culminación de la lingüística universal por Lorenzo Hervás. Ambos inspiradores a este propósito y respectivamente de Goethe y de los hermanos Humboldt, cosa aún no debidamente reconocida pero que Max Müller ya indicó en lo que se refiere a Hervás respecto de Guillermo de Humboldt, y que nosotros debemos hacer constar en la relación de precedencia de Andrés respecto del concepto de Weltliteratur de Goethe. 
A estas alturas debiera asumirse con facilidad el hecho grave, importante y usualmente eludido de que Ciencias físico-naturales y Ciencias humanas no sólo han evolucionado durante más de un siglo dándose la espalda en razón de la naturaleza ajena de sus objetos sino también de la ausencia de voluntad de relación y reconocimiento, es decir de carencia de perspectiva comparatista. Constituye ciertamente extraordinaria paradoja que unas proyecciones epistemológicas por principio comparatistas, o intracomparatistas, a la hora de establecer la posibilidad de su relación de contigüidad simplemente han hecho caso omiso, como queriendo hacer patente el carácter primordial, privilegiable e incluso displicente de un campo sobre otro. Pero nada es gratuito, y a esta gran deficiencia, cuyos matices relevantes son varios, y fácilmente reconocibles por el estudioso, y por ello mismo no nos proponemos aquí discriminar, se han venido a sumar en tiempos contemporáneos dos elementos tan perfectamente discernibles como reconocidos: a saber, el lugar común o tópico de la cómoda y, en su doble sentido, vaga, dualidad del par ciencias duras / ciencias blandas, y, de otra parte, el fenómeno efectivo y de gran alcance consistente en el definitivo establecimiento de la «serie social», la gama disciplinar de las llamadas Ciencias sociales, las cuales operan y se definen por principio en tanto posición intermedia entre los dominios seculares modernamente denominados Ciencias físico-naturales y Ciencias humanas. Pero todo sea dicho, y es evidente, las llamadas disciplinas sociales son parte principal de la disociación, históricamente natural, entre idealismo metafísico y positivismo lógico. Siendo que esto tuvo resultado, entre otros, la reconfiguración consistente en el establecimiento disciplinar de la Sociología decimonónica. A esta se debe relevantemente la marcha contemporánea de un mundo, de unas sociedades y organizaciones políticas, a las cuales directamente atañe, adquiriendo pronto la ambición de instituirse en moderno miembro disciplinar capaz de asumir, suplantar o minimizar la secular entidad de las disciplinas humanísticas al tiempo que pretendió erigirse en nueva fórmula absoluta de la ya tradicional oposición binaria, ahora denominable como Ciencias físico-naturales y Ciencias sociales, prolongando o no este último término, según conviniere, mediante el añadido «humanas». Ciertamente, esta opción ha sido no triunfante sino altísimamente divulgada, y ello no exige mucho esfuerzo explicativo como consecuencia de la manifiesta y ya señalada vinculación respecto de los modos sociales y políticos de relación comunicativa e ideologista dominantes en las sociedades contemporáneas industrializadas y, aún más, informatizadas y globalistas. Naturalmente, todo ello no es más que una grave deficiencia simplistamente camuflada de evolución modernizadora.

Se ha podido decir durante el siglo XX (quizás con notabilidad por Foucault) que las ciencias ya no se definen en virtud de objetos sino de problemas: pero esto, para hablar epistemológicamente no a la francesa sino a la anglosajona y de manera castiza, no es más que tomar el rábano por las hojas, pues todo objeto en tanto que disciplinar es concebido como problema. (En realidad, el argumento ahí convocable, pienso, es el que derivaría de un concepto de "proceso», que ahora dejaremos estar). Pero el problema de fondo que aquí late es, desde luego, el relativo a la entidad de los objetos. Si los campos y objetos físico-naturales, frecuentemente extendidos, mantienen sus objetos y las expansiones de estos ${ }^{1}$, los campos y objetos sociales, carecen de estable o sólida entidad. Y esto

1 Ello parejamente a una gran expansión de los campos tecnológicos de las ingenierías, que como es obvio en sentido estricto no definen ciencia sino aplicaciones: es por ejemplo el caso extremo actual de la Informática. En realidad, el frecuente uso en este punto del concepto de experimental no deja de ser una fórmula un tanto burda de someter a amplio examen los conceptos. La experimentalidad empírica no niega otra experimentalidad abstracta o puramente conceptual, por lo demás de mucho más evidente alcance. 
igualmente a diferencia de los objetos humanísticos, por principio de naturaleza y función no menos nítida que compleja. Aquí se sitúa en realidad el problema y en razón del cual la serie social recurre al doble apellido que suma al concepto sociológico el de objeto humano o humanístico como modo de no afrontar sino desalojar la deficiencia. Porque sin claridad de objeto, de problema, científicamente nada seguro o determinable puede haber. El objeto humanístico es evidentemente sometible a toda clase de discriminación o discusión acerca de los límites de sus entidades y extensiones fruto de la actividad del ser humano pero lo cierto es que permanece inequívoco, sustancial e irrevocable, sobre todo precisamente en su centro, no ya para la ciencia sino en general para el mundo y la humanidad: el lenguaje verbal y sus consecuentes. Ya sea esto el Verbo del Evangelio, el Quijote, el texto hegeliano, la lexicografía del sánscrito o la morfología de una gramática funcionalista. Pero el objeto sociológico difícilmente obtiene perfil estable y salida al camino de la equivocidad más allá del cuantitativismo, y este es el problema. Como es sabido, la estadistización ha venido a ser instrumentalizada como medio de legalización científica social: nada más lejos del problema epistemológico real que la efigie que suscita un volátil objeto atrapado entre estadísticas. Nótese que es en la Economía, en su serie de empréstitos entrecruzadamente matemáticos y administrativos, de contaduría y valor mercante, donde quizás la Ciencia social adquiere sentido más estable o amplia y perfiladamente razonado. Esto pudiera clarificar de algún modo el problema, pero el problema nos interesa aquí conducirlo a su posible extremo contrario, que no es sino el que erige la Educación como disciplina, y en particular, naturalmente, la Educación comparada.

El comparatismo, fundado en una modalidad mental habitual, no puede definir por principio sino un mecanismo epistemológicamente constituible como procedimiento, como método anterior o método primero, razón por la cual he insistido sucesivamente en su localización como parte de una «epistemología previa», como esencial aspecto metodológico de esta. De ahí también su universalidad, que lo es de modalidad mental y de general atingencia tanto en el campo físico-natural como humanístico y sociológico, y a su vez a la disposición histórica, geográfica y cultural de dichos campos, aun en concepto teorético y no de ciencia real. Ahora bien, sólo en Ciencias humanas y, dentro de estas en su núcleo técnico fundamental, la Filología en amplio sentido, la metodología comparatista ha llegado a adquirir la configuración disciplinar seriada; esto es, ha devenido Comparatística, y precisamente ahí de manera preponderante con este nombre. Bien es cierto que la denominación de Comparatística, de ser sometida a su plena virtualidad conceptual terminológica alcanzaría de manera natural valor de designación no ya científico-humanística en conjunto sino científica en general, pero lo cierto es que sólo ha alcanzado en ciertos sectores y países el valor común de una cierta filología y permaneciendo aún lejos de tal proceso holístico al menos en sentido explícito. Piénsese, tomando términos restringidos, en cómo la Filología Románica, por ejemplo, establecía una comunidad comparatista y no sólo intracomparatista; en cómo la Indología echaba sus bases a partir tanto de la lingüística indoeuropea, la historia de las religiones y la filosofía comparada; o en cómo la Lingüística comparada o la Literatura comparada o la Historia de las Ideas establecieron una comunidad comparatista supracomparatista, pues por principio asumían la totalidad, el mundo conocido, sobre el cual, desde luego, pero sólo a partir de ahí, operar las delimitaciones restrictivas de los objetos disciplinares 
pertinentes a la investigación concreta. Por otra parte, este holismo sectorial se complementa con una gran dimensión comparatista implícita. Resulta extraordinariamente ejemplar poder comprobar el hecho de que comúnmente las grandes realizaciones científico-humanísticas comparatistas no se autodeclaran como tales sino que sencillamente se ejercen sin manifestación programática, lo cual no deja de ser un asunto meramente formal, pero sin embargo capaz de inducir con demasiada frecuencia a gran confusión o, cuando menos, a cierto desconocimiento y menoscabo teórico. Valgan de ejemplo heteróclito para el caso la Estética y la Medicina, o la Ética y la Biología, que me permito enunciarlas sin apellidos en razón de la sustancialidad de las dimensiones con que de inmediato nos podemos referir a ellas, ya se trate de la estética aplicada o de la estética teórica, o mejor de la estética teórica del poeta y pensador José Lezama Lima y de la estética práctica del filósofo Eduard von Hartman, ambas radicalmente diversas y ambas de criterio radical y profundamente abstracto y comparatista; de la Medicina occidental holística o de la Medicina Tradicional China, o aún mejor, de la Medicina Cuántica de William Nelson; o de la Bioética y de la Biomedicina en otro orden de relación y combinatoria, capaz de consumar en el primer caso la convergencia de ciencia natural y ciencia humanística, y ciencia fundamental y ciencia aplicada en el segundo, y no sólo en el orden de la asociación de términos.

La «comparatística» en su ámbito disciplinar, el que fuere, o el «comparatismo» en tanto gama de procedimientos no pueden ser otra cosa ni por tanto definirse más que como metodología, metodología comparatista. Y en lo que se refiere especialmente al campo de las Ciencias humanas, ello viene definido como epistemología previa por cuanto pertenece por principio al mundo de existencia del objeto, a su vida irrevocable y anterior a su determinación como objeto de estudio, siendo por demás que, de no ser determinable la configuración comparatista, o bien nos encontraríamos ante la atenuación o pérdida de fundamento epistemológico propiamente dicho o bien ante objetos mal constituidos, de determinación mal formulada ${ }^{2}$. Por lo demás, una novedad creciente en los últimos tiempos consiste en la articulación de aquello que podemos denominar no tanto objetos compuestos, aunque también, sino disciplinas compuestas incluso en alto grado de distancia o diferencialidad, así el caso de la Bioética, sobre lo cual no nos interesa ahora entretener argumentos.

A las disciplinas físico-naturales corresponde un comparatismo objetualista en el sentido de no regido por la completa esfera del sujeto tal como acontece a los objetos humanísticos, según de hecho venía a formular la epistemología de Dilthey y su penetrante especificación del objeto humanístico como sujeto que se autoobjetiva para ser devuelto a su objetividad que no es sino ser del propio sujeto. Evidentemente el mejor ejemplo sería el lenguaje verbal, es decir el objeto humanístico primordial. A partir de ahí cabría proponer la clasificación del comparatismo de las disciplinas físico-naturales como, preferentemente, «comparatismo extrínseco», frente al preferente «comparatismo

2 Como anteriormente aduje, he insistido con cierta frecuencia y en términos generalizables para la serie de las Ciencias humanas (por ejemplo en «Epistemología de la Teoria y la Crítica de la literatura», Teoría de la Crítica literaria, Madrid, Trotta, 1994, p. 22), acerca de que «el comparatismo no constituye una mera opción metodológica o disciplinaria sino que es imprescindible a todas ellas, las entrecruza, pues se encuentra en la propia base dc toda actividad crítico-literaria por cuanto que viene inesquivablemente especificado por cl mundo de existencia del objeto, ante el cual sólo cabe la aceptación de hecho. Por ello, el comparatismo (...) pertenece a la epistemología crítica previa, al fenómeno de hacerse patente la propia constitución de la obra literaria y la probabilidad de acceso a la misma como objeto crítico bien constituido». 
esencialista» científico-humanístico. Siendo que en ambos casos es de idéntica aplicación tanto la dualidad tipológica de comparación «de facto» o comparación "por analogía», al igual que de otra parte el aspecto de «distancia» como principio cuyo factor de incremento presupone positiva virtualidad comparatista. Esto es, a mayor distancia de los términos u objetos de comparación, mayor virtualidad comparatista. Todo lo cual hace patente, aun si cabe en mayor grado, la entidad relacional o de términos de la comparación, inherente, aun en la convergencia más natural e íntima, a toda metodología comparatista. Aquí es donde existe una evidente divergencia humanística y físico-natural, en razón, dicho resumidamente, del dominio del factor cultural histórico y geográfico del objeto humanístico frente al factor material del objeto físico y experimental.

$\mathrm{El}$ aspecto extrínseco físico-natural del objeto lo es en tanto externo al sujeto humano, sujeto como consciencia (consciencia inetelectual, verbal, emocional...), sin que esto deba conducir por otra parte a la creencia en esa suerte de ficcionalismo empírico de la ciencia que cree en una existencia sin relación al sujeto. Podrá tratarse de una relación lo más restringida posible respecto del sujeto, en virtud de la exactitud cuantitativa o ecuacional, de la generalización física o químicamente legislativa y ahistórica, pero en cualquier caso no existe observación sin observador ni observador fuera del tiempo, la historia de la cultura y la ciencia. En fin, por llegar a la obviedad, no existe ciencia sin sujeto, y esta en todo caso debe ser definida como la mayor capacidad y efectividad posibles de rigor en el tratamiento del objeto. Y siendo toda premisa, en principio, no más que la declaración programática y sus razones técnicas o teóricas, y éticas.

Junto al denominado método científico por antonomasia de las disciplinas físiconaturales, su gama (experimental, hipotético-deductiva, y de medida compleja y no sólo cuantitativista), e incluso las posibilidades transicionales respecto de las disciplinas humanísticas (así los métodos analítico y observacional) y las sociales (así característicamente las modalidades estadísticas y cuantitativistas) las Ciencias humanas y las sociales han de razonar su propio estatuto y clasificación. A todo ello queda convocado un nuevo invitado digital y computacional, fundado (nunca se olvide) en la ingeniería informática.

Que la metodología comparatista es fundamental a toda constitución del conocimiento, de las ciencias en general y las humanas sobre todo, es quizás cosa tan evidente como pocas veces explicitada de manera taxativa, y es por ello que nosotros lo debamos reiterar y subrayar. Es infrecuente o escasa, e incluso hasta en ocasiones de consecuencia equivoca, la producción teórica, o meta teórica por mejor decir, de argumento comparatista $^{3}$. La respuesta a esta constatación se diría aparentemente sencilla y relativa a una mera coincidencia epistemológica sectorial, pero 1a reflexión en torno a las diferentes disciplinas y la Literatura comparada en particular hace patente en este sentido un resultado un tanto más complicado, envuelto quizás entre la especialización disgregadora, por una parte, y, por otra, las singulares circunstancias que durante la segunda mitad del siglo XX y hasta hoy han definido la cara institucional o académico-administrativa de ese campo literario, no pocas veces manipulado, y esto desde luego no siempre en el sentido de una simple y legítima orientación íntelectual4 ${ }^{4}$ E1 problema disciplinario del

3 Cuando, por una vez, fue planteada la cuestión comparatista a propósito de las Ciencias humanas en general (por Jucquois en 1989), no se supo la importancia que esta poseía asimismo para las ciencias físico-naturales ni, lo que en su caso resulta aún más defectuoso, la decisiva importancia del "parangón” grecolatino en la historia del humanismo, la filología, la literatura y en general la cultura clasicista europea.

4 Es por otra parte observable cómo el propósito de una u otra manera enarbolado por algunos, a fin de aquilatar su posición, de que la Literatura comparada era, no ya propiamente una disciplina en sentido fuerte (idea que con el tiempo hubieron de abandonar por exigencia contextual del «guión epistemológico» para hablar de situaciones institucionales académicas) sino un campo en realidad 
comparatismo humanístico, durante el pasado siglo distintivamente enarbolado en el campo de 1a ciencia literaria, ha estado aquejado por el abuso de cierta política y defensa de intereses nacionales y culturales. Este tópico interno de la historia de la Literatura comparada, curiosamente ha sido con posterioridad reconstituido con diferentes elementos y propósitos, que no por hábilmente enmascarados habrá que aceptar.

Es preciso subrayar, como dijimos, que si todo comparatismo no es sino metodología, por consiguiente quienes no han formulado una consecución del método o una epistemología, disciplinaria o histórica, como metodología de la comparación misma, no han actuado sobre el núcleo y fundamento teórico del problema.

La relación de universalidad, globalización y comparatismo, que son o debieran ser términos indisociables del pensamiento de nuestra época, significa nuevamente de manera inevitable, o recupera en otros casos, una intromisión en el terreno de las ideologizaciones, intromisión expansiva que ya se ha podido apreciar con caracterizaciones propias de nuestro tiempo mediante el concepto, erróneo, en sentido propio, de «multiculturalismo», concepto inconsistente sobre el cual a estas alturas no merecerá la pena dilatar aquí argumentos. Sólo el desconocimiento del carácter y la función de los valores culturales puede conducir a la asunción de una tal doctrina, contradictoria en sí misma,

He sostenido en otras ocasiones que la globalización ha de concebirse como una universalidad cuya disposición al tiempo esencialista y orgánica del hombre y el mundo cuyo sentido se obtiene de las consecuciones de la adecuación entre la unidad y el todo. Esto es, la unidad no es lo contrario de la pluralidad sino el principio mediante el cual esta se obtiene o muestra como un todo. Por ello la universalidad es revelación del espíritu y su aspiración se comprende plenamente en tanto que referida al ser humano todo y la humanidad íntegra en su pleno sentido posible. A diferencia de 1a globalización, en su ejecución real ceñida a un régimen internacionalista de mercado al amparo de la extremada velocidad de la comunicación electrónica y la progresión de los transportes, la universalidad ha de ser concebida justamente en auténtico sentido humano. La universalidad no sólo antecede sino que ha de subseguir a toda globalización, si la humanidad no fracasa, pues se refiere a la comprensión y la fuerza del ser humano y las culturas. El grave problema no afrontado por la globalización, o intencionalmente omitido, es el de la homogeneización de las culturas, así como el resorte del aprovechamiento de lo peor de casa.

Existe una gran dificultad al parecer aún creciente y no sabemos si definitivamente fuera de control éticamente fundado. Me refiero al muy intenso escenario actual de sectores seudodiscíplinares fundados en la ideologizacíón. Pero la universalidad comparatista se encuentra a la base de todo saber y toda práctica humanística. Entiéndase, ese diagnóstico no refiere sino uno de los factores operantes, aunque sí el último y por ello más concernido, en el proceso contemporáneo de desintegración o agresión a los campos humanísticos decisivamente facilitado por la destitución rectora de la Filosofía, a lo cual desde un principio aquí nos hemos referido intentando evitar toda cómoda simplificación.

creado fundamentalmente a partir de la última guerra (transponiendo así la situación norteamericana al resto del mundo), ha tenido, fuera previsto o no por aquellos sus ideadores, éxito en las últimas generaciones universitarias, de entre las cuales ya no es fácil la localización de estudiosos cuyo horizonte intelectual sostenga algún despliegue notable en el tiempo de la ciencia y el saber. El hecho es que este fenómeno, asentado por demás en importantes sinergias, se ampara a1 tiempo que fundamenta esa ingente falacia actual formulada en términos de que la nuestra es la «sociedad del conocimiento", confundiendo información y reflexión. Ello confluye en una depredación de las Ciencias humanas por desgracia promovida vehicularmente, como no podía ser de otro modo, gracias a pujantes sectores sociales y académicos inicialmente originados en lengua inglesa. 
Es un grave problema y aún está por dirimir una salida lo más correcta posible a la destitución filosófica y, acaso, también filológica ${ }^{5}$. Aquello que sintéticamente podemos denominar 'sociologización' constituye el sesgado fenómeno de nuestro tiempo que en buena medida rige esta problemática, de la que asimismo forma parte la Educación.

\section{III}

La Educación presenta el aspecto doble de disciplina humanística, de objeto humanístico, y de disciplina social cuyo objeto queda sobrepuesto al anterior, cada vez más reducido en los campos de decisoriedad académica, pues no se olvide que la Educación apenas tiene existencia fuera del ámbito académico, ámbito que fundamenta y sostiene pero también retroalimenta y a veces parasita, en el peor de los casos. De ahí la noción que permite resumir este problema como de «padecimiento pedagógico». Uno de los problemas más espinosos de la disciplina educativa consiste en su anteposición metodológica a las disciplinas de contenido o de efectiva materia. Es decir, la Educación se funda contemporáneamente como Didáctica y como Propedéutica y de hecho lo hace a menudo al margen de materia alguna más allá de su ámbito previo a toda materia. Aquí hay un problema de conexión mejor o peor resuelto según las diferentes disciplinas particulares.

El caso de la Educación y en particular de la Educación comparada permite efectuar alguna ejemplificación contrastiva notablemente iluminadora a fin de contextualizar los campos disciplinarios significativos por entrecruzados o de doble aspecto. A diferencia del estable predominio disciplinar humanístico de la Filosofía y sobre todo de la Filología, y a diferencia del perfilado objeto sociológico de la Economía, la Educación describe una evolución moderna tendente a la cesión humanística en favor de la constitución sociológica. Pese a todo, es de afirmar que el ámbito disciplinar de la Educación permanece en términos teóricos centralmente humanístico en virtud de la definición de su objeto central o esencial. Ahora bien, la Educación también define, en nuestro tiempo, un objeto humanísticamente sólo secundario, pues al objeto primero se sobrepone su aplicación al estudio de la Educación no en sí, internamente, sino de manera extrínseca, en tanto que sistema educativo y en tanto comparación externa de sistemas educativos, comparación que adquiere su mejor virtualidad, como es evidente, en la comparación internacional e incluso el redimensionamiento producido por la formalización del Plan europeo denominado Bolonia. El asunto permite, ciertamente, mayores especificaciones, pero no serán

$5 \quad$ No quiero dejar de advertir, hablando al margen de los caídos neopositivismos estructural-formalistas del siglo XX, acerca de la fuerte agresión de las administraciones europeas respecto del futuro de las Ciencias humanas y el humanismo en general (puede verse sobre ello el primer capítulo y en alguna medida los tres primeros volúmenes de nuestra Teoría del Humanismo, Madrid, Verbum, 2010, 7 vols.). Ello al amparo de la excluyente epistemología popperiana y de la malversación disciplinar de Roman Jakobson, pero sobre todo no quisiera dejar de advertir acerca del peligroso silencio que desde la situación actual, un tanto acosada y depauperada, de las ciencias humanas se viene manteniendo frente a la gran presión tecnológica y economicista, tanto en la esfera política como académica y en la intersección de una y otra. Porque precisamente en esa presión tecnológica, amparada en un entendimiento finalista del mercado en connivencia con ciertas y evidentes concepciones sociales y políticas acríticas y en realidad trasnochadas, es donde reside la mayor responsabilidad internacional del estado actual lamentable de la distribución del trabajo y la riqueza, de la inestabilidad de las estructuras económicas y de los mercados, de la profunda falta de inteligencia con que esto ha sido afrontado durante el último medio siglo, siendo ello en principio predominante responsabilidad occidental como visión sociopolítica del mundo. Esta situación en la que ha despertado el siglo XXI no es desde luego resultado de la actitud ni de la aplicación humanística, como algunos ahora amparados en la nueva operatividad del poshumanismo y la posverdad quieren hacer creer, sino resultado de la paulatina postergación de las posibilidades de aquella opción humanística. 
necesarias para lo que ahora me propongo. He ahí el fundamento comparatista de razón suficiente para la Educación como disciplina de la serie social y no sólo humanística. Diferente cuestión es la relativa al examen epistemológico concreto del caso y su posible pormenorizada valoración, que aquí no afrontaremos.

Pienso que de formar una razón histórica y teórica más general y moderna que la representada para la Educación, en cierto modo simbólico, por Comenio, esta ha de ser fuertemente metodológica y encuentra su dualidad, dicho en términos igualmente resumidos y un tanto simbólicos, en los pares de definición social y humanística Comte-Durkheim y Schleiermacher-Dilthey ${ }^{6}$ Y quiero decir, para este segundo caso, la Educación, en tanto que humanística, ha de retrotraerse al objeto verbal, sin el cual nada hay, tanto esencial como aplicativamente.

El momento actual, en tiempos de Globalización, del campo disciplinar educativo, no se olvide que de algún modo se va a ver obligado a recentrar el objeto humanístico clave del sujeto de aprendizaje, la lengua y la lectura. Ello por la sencilla razón de que en torno a la lectura, su frecuente fracaso, y de otra parte la instrumentalización política de las lenguas como medio de intervención educativa en algún grado opresiva se ha creado un gigantesco problema que habrá de emerger en su descomunal dimensión hoy por hoy medio sumergida. Pero por otra parte, también es de recordar el hecho de que la formulación comparatista actual de la Educación, es decir la Educación comparada, pasa necesariamente por la comparación de los términos Asia/Occidente, y esto no ya como relación internacional sociológica o sociopolítica sino también y sobre todo en su aspecto esencial interno. Nada mejor para ello que una invitación a comenzar con la necesaria lectura del texto Sobre la Educación de Aurobindo, quien con nitidez delinea a este propósito las fuerzas del contraste de las dos grandes civilizaciones matrices.

El presente monográfico comparatista que tiene ahora el lector a disposición, se caracteriza por la brevedad y una propuesta esperanzada en la eficacia y la concreción, tanto epistemológica como histórica, tanto aplicativa como metodológica. En lo que se refiere a esto último he preferido solicitar un caso muy particularizado mediante la música desde el punto de vista educativo, así como una muestra metodológica en el campo de la Ciencia literaria, probablemente el ámbito contemporáneo de mayor proyección comparatista junto a la Historia de las Ideas. He preferido aprovechar la ocasión que generosamente nos ha brindado la Revista Española de Educación Comparada, por mediación de la profesora $\mathrm{M}^{\mathrm{a}}$ José García Ruiz, no para desplegar un extenso proyecto pluridisciplinar sino a fin de proponer un breve y paradigmático mosaico de Educación comparada mediante muestreo original. Nueve artículos no extensos sobre materias y desde perspectivas poco comunes y por otra parte actuales son suficientes para poner de manifiesto nuestra propuesta que, como he dicho, puede complementarse con la problemática de la lectura, a la que ya me he referido con cierta extensión en otro lugar, y la lectura hoy accesible del ensayo pedagógico de Aurobindo. En fin, el presente monográfico se quiere modesto complemento comparatista del trabajo desarrollado por el profesor José Luis García Garrido en la fundamentación de la Educación comparada.

6 Es preciso a mijuico recuperar desde el ámbito educativo la disciplina hermenéutica como caracterizadora metodológica de las Ciencias humanas y toda vez que ofrece de manera integrada, como metodología, según la terminología antedicha, interna, el comparatismo, cosa por lo común, hasta donde se me alcanza, no advertida. El método hermenéutico, la comprensión centrada por el teólogo romántico Schleiermacher para el ars interpretandi, se aloja en orden a1 horizonte de la Dialéctica y consta de dos aspectos o momentos en todo caso finalmente sujetos a circularidad: el comparatista y el adivinatorio, y aun este último, habría que recordar, en remisión a su vez a la comparación con uno mismo, del intérprete consigo mismo. Puede verse el volumen de la reciente edición y estudio de la Hermenéutica completa de Friedrich Schleirmacher preparada por Rosario Martí Marco para el Instituto Juan Andrés. 